\title{
Study on Computer Technology and Information Management Based on Integration Perspective
}

\author{
Zhangkui Liu \\ Baoshan University, Baoshan, 678000, China
}

Keywords: PIM, Integration, Computer technology, Information management, Use intension, Logic

\begin{abstract}
In current era when mobile internet and computer information technology prevail, every individual is information communicator and receiver in society. In the face of rapid social development and huge scale of information communication, human beings depend on computer information management technology and relevant assistant management tools to conclude, integrate and utilize information, which has become a normal situation. This paper takes personal information management tool PIM as the subject and discusses computer PIM integration oriented to virtual and physical property and its specific deign application.
\end{abstract}

\section{Introduction}

Computer PIM technology becomes an indispensable part of human life, because current social development becomes increasing "information fragmentization". Regardless of development of society and social individual, communication of countless information resources from all directions has becomes a normal life state. Thus, human beings need to adjust thinking, expand view and integrate advantageous information resources with long-term development mentality so as to gain development rhythm which complies with social rules and reflect reality and innovation of computer technology and information management for human development based on the integration perspective.

\section{PIM}

\section{Overview of PIM}

PIM, also called PIMS, is Personal Information Management System. The original design concept of PIM was proposed by American scientist Vannevar Bush in 1945. It can be regarded as a computer technology assisting human memory expansion. It was initially used in personal documents and libraries in America, and named memex information management system. It can be seen that as electronic computer appears and information communication in society becomes increasingly mature, some considered effective management of massive technical information which might be brought by scientific development and especially processing of personal information as early as the middle of last century. In 1961, some proposed problems related to personal information processing. Then, "Microdocumentation System" appeared. It can handle and manage personal records and modification in books and files. Based on this "Microdocumentation System, American scientist Engelbart made future predications. He considered computer technology and information management platform in future society should gradually tend to automation, intelligence and efficiency. Thus, management system based on information resource integration must be established to relieve the pressure to human development caused by massive information communication when personal information environment develops rapidly. In 1988, another American scientist Mark Lansdale published an article in Applied Ergonomics. The article mentioned "PIM psychology". PIM appeared in human society the first time. The article indicated that enterprise office in future society should provide office staff with effective and sufficient information management system and provide information retrieval mode together with the system. This imagination also reveals human strong desire to bid farewell to paper media society and develop to the era of science and technology. In recent years, since technological developmental level and human production and life needs improve 
increasingly, PIM research has received more and more attention, and the research range of PIM also become wider and wider. European scholar Bergman in computer technology field defined PIM. In his opinion, when users apply computer to manage personal information such as email, document and contact person, their aim is to plan their daily work, life and leisure-time activities. Therefore, he believes the ideal state of PIM is "computer users can always find out correct information at the correct position, and improve quality through various compatible formats to meet users' real-time demand. ${ }^{[1],}$

\section{Main activities of PIM}

PIM as an important technical idea of personal information management in modern computer technology mainly participates in discovery and search activities. In particular, meta-level PIM activities boost its information management quality and human work efficiency.

\section{Search activity of PIM}

American computer scholar Jones indicated, "the activities PIM contains are related to new information acquisition. It may be out of occasionality and inevitability of human awareness. But its final aim is to organize and conclude information to the specified storage space by computer activity for the convenience of personal information re-search and re-utilization". Thus, search activity is an important function of PIM system.

As the process of personal information management, PIM behavior will generate interaction relationship with other fields of science. Hence, with the rise in personal information amount and continuous technological development, the definition of PIM behavior also has great extension possibility. For example, current PIM activities are closely related to computer science, ergonomics and psychology. Therefore, search activity increasingly becomes an important behavior of PIM system. It humanizes personal information management and its relevant behavior operation from the perspective of psychology, and shows the progress of information management technology.

Acquisition activity of PIM

Acquisition activity of PIM is mainly divided into two parts: active and passive information acquisition. Active information acquisition refers to information acquisition behavior based on the precondition where users own certain information consulting ability, so active acquisition can be divided into information cognition, creation and re-creation processes. Information re-creation is advocated by social information development. It reprocesses raw information, digs new value and boosts existing information system ability.

Another is passive information acquisition which can also be understood as human unconscious information acquisition process. In human work, learning and life, some information is known by people through different channels and forms memory in brains. The invention of penicillin is taken for example. Its creator Fleming unconsciously cultivated staphylococcus in a dish lid due to his negligence in medical experiment. As a result, cyan mycete growing on the lid killed all staphylococci. Fleming occasionally found such important information and studied penicillin later. A typical passive information acquisition is described here, but passive acquisition also needs to own certain information discovery ability.

PIM activity at meta-level

PIM activity at meta-level refers to an orderly management activity in allusion to personal information space and set. It combines metadata reprocessing to form new data with higher value, so it is called "data management in data" in computer field. According to this title, PIM can be understood as a comprehensive management activity which makes information gives play to its function through maintaining PIM overall data management framework with meta-level activities, as shown in 1. 


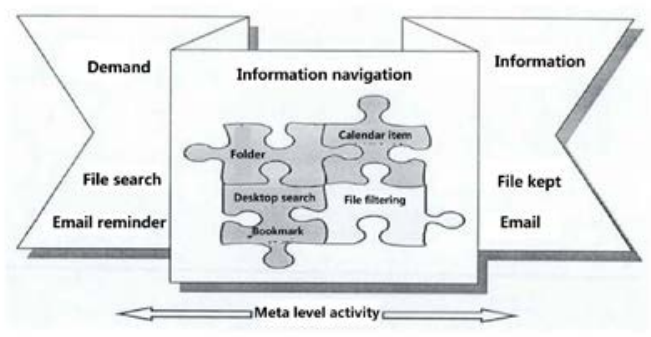

Fig.1 Schematic diagram of information integration management activity at PIM meta level

Fig.1 shows information management activity of PIM in each meta level. The middle part is PIM mid-meta level. In this level, information acquisition and storage behaviors happen, so it has overall attribute relative to all activities at the whole meta level. If retrieval engine is utilized to inquire and store information at meta level, overall efficiency of PIM activities will be affected. In people's actual application, PIM often integrates email, electronic document and bookmark functions. At this moment, activities at PIM meta level will launch some storage and retrieval functions to assist artificial information management behavior. Such humanization design brings convenience for human production and life. So, PIM tool as a computer information management technology receives more and more attention.

\section{Concept and design application of integration-based PIM system}

\section{Basic concept of PIM oriented to integration perspective}

Like most computer information management technologies, PIM system oriented to integration perspective concludes multiple functions and utilizes integration method to rationally manage computer information functions such as email, operation interface and communication re3cords. Under the planning of PIM system, personal integrated information system is established, and all kinds of information are correlated through respective nodes. This standardizes and promotes personal information management efficiency. In Britain, intelligence scholar Brooks utilized PIM to conclude and manage documental information he collected, finally evolved to knowledge management, applied it in libraries of various universities to form systematical knowledge network structure and to become "knowledge map" structure upheld by British society. In the opinion of Brooks, literature arrangement from physical level to technological level is transformed to knowledge unit. Such transformation behavior is intelligence unit transformation. With the help of PIM system, knowledge information is transformed to pragmatics level from grammar level. This means personal information of multiple individuals establishes correlation. Finally, certain scale of knowledge relevance forms. Effective management mode based on uniform interaction forms through PIM system. So, Brooks's practice laid a foundation for future development of PIM ${ }^{[2]}$.

PIM system design oriented to integration perspective

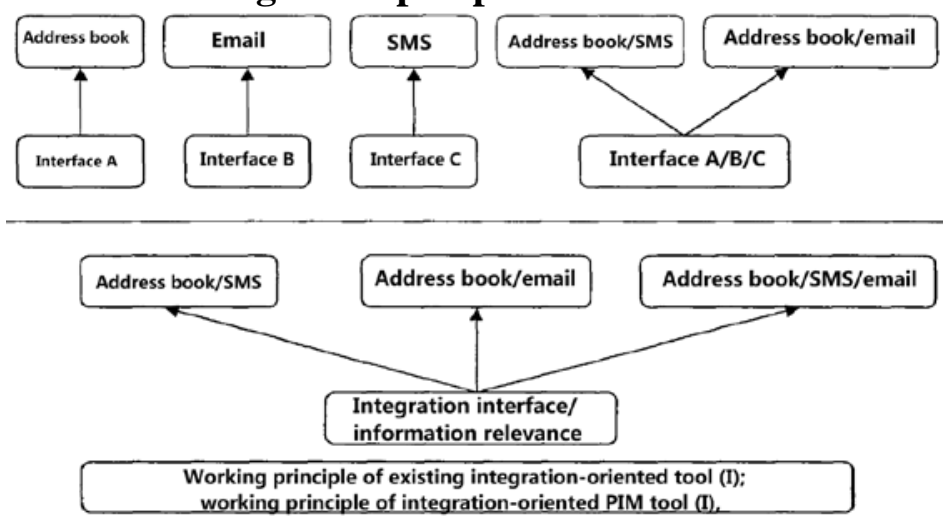

Fig.2 Structure diagram of PIM system design oriented to integration perspective 
It is known from Fig.2 that PIM system design mainly aims at single type of personal information management platform. As shown in the upper part of Fig.2, Interface A is responsible for address book management; Interface B is responsible for email management; Interface C is responsible for SMS management. They are typical single management design. Thus, users can only use one function within single time bucket. In practical operation process, such situation greatly influences work efficiency. Hence, as shown in the lower part of Fig.2, after improvement, management interface based on function integration supports multi-functional simultaneous operation management. When users operate email, they can operate SMS sending at the same time. PIM based on integration perspective greatly improves in terms of assisting personal information management. Any.do is dominated by schedule management. It can help users to manage personal communication information and integrate all software tools. Tasks is mainly responsible for task management. The concept of integration perspective offers multiple possibilities of information management and application for PIM system. Meanwhile, it also enriches personal information management types. More importantly, it can dig information relevance node among personal information according to foundation of original system and find out implicit relevance among different types of personal information. This is also the key to boosting users' management of personal information efficiency.

\section{Specific application of personal information integration in PIM system based on virtual attribute}

PIM system based on integration perspective owns traditional personal information integration mechanism with physical property, but with technological development, construction of personal information management from physical property to virtual property has become the general trend. Thus, Professor Dawn.Lawrie from Massachusetts Institute of Technology studied the prototype of "self-organizing filing cabinet" paper document information management. It improves information management quality of paper media through three steps: scan - transform data - store. There are many similar virtual information management technologies, such as integration of webpage bookmark and email.

Webpage bookmark integration

PIM system based on integration perspective has its uniqueness in persona webpage information management. It can help computer users integrate webpage browsing records in recent 10 times and classify webpage information. Such principle is applied in tree-shaped visual interface, footnote and thumbnail planning mode, improves network browsing efficiency of personal users, integrate and classify webpage information automatically according to users' habits. 2D window, Integrated System thumbnail and Bookmark historical bookmark are based on PIM system and combine 3D visualization technology to integrate and manage webpage information. In essence, such humanized scientific classification is totally based on webpage integration prototype - Personal Web. This prototype owns strong functions in terms of hierarchical structure, such as multi-document, multi-webpage and multi-bookmark integration and management. It establishes connection through internal code of webpage and offer high-quality and efficient webpage information integration management services for users. It is a network information management mode all will adopt worldwide.

\section{Email integration}

Email is an asynchronous information communication tool which bears the most PIM functions. Branch system of SwiftFile prototype exists in PIM system based on integration perspective. It can carry out structure layering for personal email folder to reach the purpose of managing, integrating, concluding and classifying email. It can even detail all information in email including information of dialogue, attachment and contact person. The prototype of Taskmaster manages email information integrated in interactive interface. It first layers email in interactive interface, such as three layers: email list, attachment content and email information. This contributes to the system to help users uniformly integrate key points in information. Personal schedule, backlog, document transmission and handling in email depend on PIM system for integration and management. For commercial users, it is a very humanized and convenient information management mode $\mathrm{e}^{[3]}$. 


\section{Conclusions}

In one word, PIM system based on integration perspective has developed to certain level in terms of integrating cross-functional and cross-type information, so it is widely applied. Its safety, perception effectiveness, usability and stability have been approved by the industry. "Knowledge map" idea it advocates is also a trend in computer technology and information management research and owns active reference value for future computer PIM system development.

\section{References}

[1] Hou Ming, Liu Yang, Integration of computer application technology and information management. Net Friends Cloud Education, 2014,(6):19-20.

[2] Wang Wentao, Xie Yangqun, Li Jing et al. Empirical study on use intension of integration-oriented PIM software tool. Information and Documentation Services, 2013,(6):41-46.

[3] Wang Wentao, Study on integration-oriented PIM tool. Anhui University, 2013.44-52. 